\title{
Hemispheric asymmetries in the cortical evoked potential as a function of arithmetic computations
}

\author{
CHARLES T. RASMUSSEN, ROY ALLEN, and ROBERT D. TARTE \\ University of Nevada, Las Vegas, Nevada 89154
}

\begin{abstract}
Hemispheric asymmetry in the cortical evoked potential (CEP) was examined by means of a distracting task involving mental calculations. A considerable body of evidence suggests that CEP amplitude is, at least in part, positively related to attention to the evoking stimulus. Also, a growing number of studies suggest that arithmetic computation is primarily a function of the dominant hemisphere. In the present study a task involving mental arithmetic was used as a distraction. Results supported the hypothesis that the experimental task would produce greater attenuation of CEPs from the dominant hemisphere.
\end{abstract}

The findings of such investigators as Milner (1965) and Sperry (1968) have provided a considerable body of evidence to support the hypothesis that the human cerebral cortex is functionally laterally asymmetrical. In the course of the last several years, a number of efforts have been made to better understand the electrical nature of this asymmetry through the use of recordings obtained from scalp electrodes. These data have taken the form of both electroencephalograms (EEGs) and computer-processed cortical evoked potentials (CEPs).

A number of investigators have examined EEG records obtained simultaneously from both left and right hemispheres with the goal of detecting asymmetries resulting from tasks assigned to the subject. The most prominent and consistent finding has been that when the subject is task involved, alpha is bilaterally suppressed or attenuated and that this effect is most pronounced in left-hemispheric recordings in the case of verbal or computational tasks and in right-hemispheric recordings in the case of spatial tasks (Butler \& Glass, 1974; Dumas \& Morgan, 1975; Lorens \& Darrow, 1962; McKee, Humphrey, \& McAdam, 1973; Morgan, McDonald, \& Hilgard, 1974; Morgan, McDonald, \& Macdonald, 1971). Of these, McKee et al. (1973) found that the effect of left suppression as a function of verbal task becomes more pronounced as task difficulty increases; they also reported that musical tasks serve to attenuate right-hemispheric alpha. Pattern recognition and manipulation also appear to accentuate the hemispheric asymmetry, with these tasks tending to decrease right alpha (Dumas \& Morgan, 1975).

This research was supported in part by the University Research Council, University of Nevada, Las Vegas. Portions of the material presented here have been presented at the spring 1977 meeting of the American Association for the Advancement of Science. Portions of the research reported here were part of a master's thesis submitted to the Department of Psychology, University of Nevada, Las Vegas, by the second author.
In a somewhat different approach to EEG analysis, Galin and Ornstein (1972) examined ratios of righthemisphere to left-hemisphere whole-band power $(1-35 \mathrm{~Hz})$ from temporal and parietal leads. These ratios were found to be markedly higher for verbal than for spatial tasks. In a subsequent study, Doyle, Oinstein, and Galin (1974) performed a frequency analysis of the above-mentioned power ratios; the power asymmetries were found to be most prominent in the alpha band and less consistent for beta and theta bands. In the delta band, no consistent effects were found.

In CEPs obtained simultaneously from left- and right-hemisphere electrode placements with right-handed subjects, verbal stimuli result in greater amplitude at the left hemisphere and certain nonverbal stimuli result in greater amplitude at the right (Buschsbaum \& Fedio, 1969; Cohn, 1971; McAdam \& Whittaker, 1971; Morrell \& Salamy, 1971; Neville, 1974). In addition, Neville (1974) reported that verbal stimuli also result in shorter latencies from left-hemisphere placements. When used as distractors, verbal and spatial tasks result in reduced amplitude from left-hemisphere CEPs evoked by a nontask stimulus (Galin \& Ellis, 1975). Davis and Wada (1974) examined coherence or similarity of form of CEPs obtained from temporal and parietal leads from the same hemisphere; they reported that clicks result in CEPs of maximal coherence on the speech-dominant side, while flashes result in greatest coherence on the non-speech-dominant hemisphere. Musical chord stimuli have been shown to result in greater CEP amplitudes at the right hemisphere (Taub, Tanguay, Doubleday, Clarkson, \& Remington, 1976).

In another body of CEP literature, it has been shown that attention to, or distraction from, the evoking stimulus serves to modify the amplitude and latency of the CEP (Ciganek, 1964, 1965, 1969; Garcia-Austt, Bogacz, \& Vanzulli, 1964; Haider, Spong, \& Lindsley, 1964; Spong, Haider, \& Lindsley, 1965). Attention to the 
evoking stimulus tends to result in increased amplitudes and decreased latencies, while distraction, due to an assigned task or another stimulus, produces the opposite results.

The research reported below is an attempt to further examine the electrical nature of cortical asymmetry by using a task involving mathematical calculation as a distraction from an evoking visual stimulus. Based on the reports of the asymmetrical effects of computation tasks on the ongoing EEG, it was expected that amplitudes from left-hemisphere CEPs would demonstrate a significantly greater degree of attenuation during a distracting computational task.

\section{METHOD}

\section{Subjects}

Twelve subjects (eight male and four female), aged 16 to 28 years, served. All subjects were right-handed, with no history of brain damage. Benton (1965) reported that for right handed subjects the probability of left-hemisphere dominance was quite high.

\section{Apparatus}

EEGs were obtained from monopolar electrodes (silver) at C3 and C4 (Jasper, 1958) referenced to the ipsilateral ear; a chin electrode was used for grounding. These signals were amplified using a Grass Model 7 system and two Grass 7P511 EEG amplifiers. A Grass PS22 photo stimulator was used for stimulus presentation with the intensity setting at 16 . Signal averaging was accomplished by means of a Digital Equipment Corporation Lab 8e-25 computer.

\section{Procedure}

For both control and experimental conditions, the subjects were seated, eyes closed, facing the strobe at a distance of $5 \mathrm{ft}$. The stimulus was a double flash with a $150-\mathrm{msec}$ interflash interval; presentation rate was one stimulus per 2 sec. The CEP sweep was initiated with the first flash of the pair and continued for $500 \mathrm{msec}$. Each average was based on 50 sweeps. In the control condition, the subjects were instructed to detect a "third flash, which may or may not occur during this run." For the two experimental runs, subjects were instructed to count backward from 600 by sixes and from 700 by sevens, respectively. The experimental and control runs were arranged in an ABBA fashion for half of the subjects and BAAB for the other half, resulting in four pairs (one left and one right) of averages per subject.

\section{RESULTS}

The data from a typical subject are shown in Figures 1 and 2 . This represents only one-half of the data from this particular subject since two averages (left and right hemisphere) were obtained from each subject on each of four runs, two experimental and two control.

In all CEPs, the most prominent component was a biphasic wave with a negative peak in the range of 75 to $150 \mathrm{msec}$. The peak-to-peak amplitude of this component was obtained for each CEP. For each run, a ratio of right amplitude to left amplitude was calculated. The two ratios for the experimental runs were added together, as were the two ratios for the control runs; the

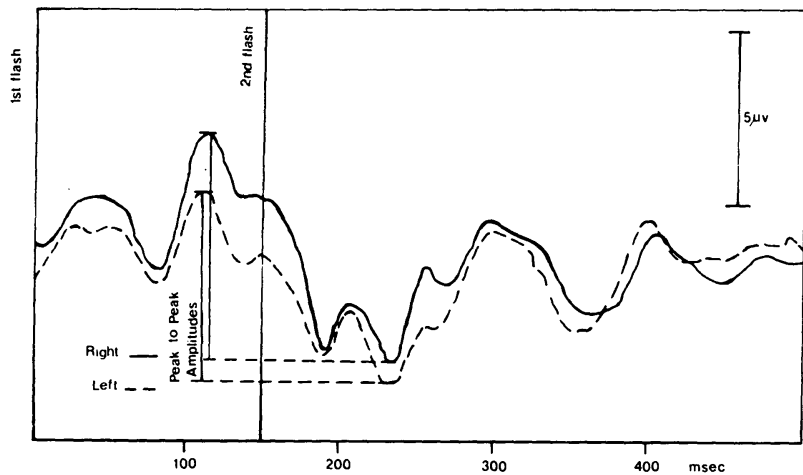

Figure 1. Average evoked potentials from Subject 5 during an experimental run. Upward deflection corresponds to negative at the active electrode.

control sum was then subtracted from the experimental sum. The remainder was a positive value if the lefthemisphere amplitude decreased more than the right during the experimental runs as compared to control runs; it was negative if right amplitude decreased more.

Examination of the data reveals that the experimental task produced significant left-right amplitude differences in the CEP when compared with the control task ( $p<.05$, Wilcoxon matched-pairs signed-ranks test), with the shift being in the direction of reduced lefthemisphere amplitude during the experimental task, as predicted. No consistent latency shift was found as a function of the experimental task.

\section{DISCUSSION}

The results reported here provide further support for the hypothesis of functional cortical asymmetry. More specifically, they support the contention that computational functions involve the left or dominant hemisphere in right-handed individuals. The left-right CEP asymmetry may also be explained in terms of slight differences in amplifier settings or in terms of bilateral electrode placements which were not homologous. In the present situation, considerable care was used in calibration and electrode placements. Furthermore, the differences reported

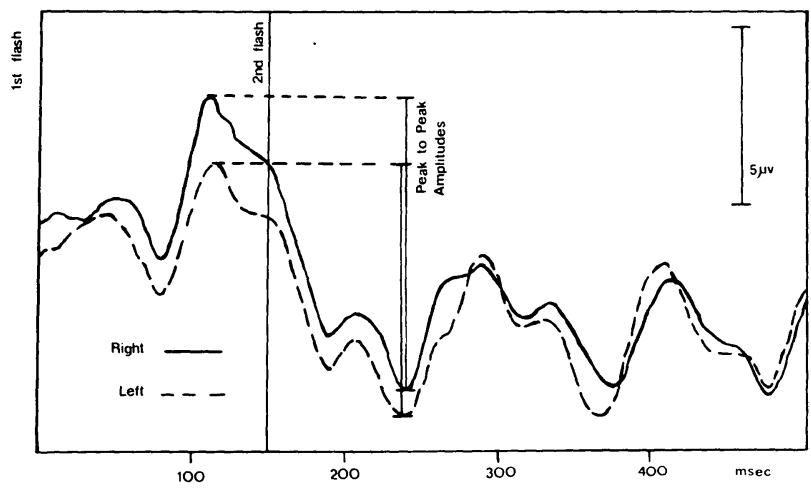

Figure 2. Average evoked potentials from Subject 5 during a control run. Upward deflection corresponds to negative at the active electrode. 
are based solely on within-subject shifts due to manipulation of the experimental condition.

Many of the studies reported have found increased amplitude from the task-involved hemisphere. However, in all of these studies the evoking stimulus was task relevant. In comparing our findings with them, it is well to emphasize that, in the present experiment, the task of interest (computation) was used as a distractor, while the CEP is based on a non-task-related stimulus (flash). In this situation we predicted that the task-involved hemisphere would reflect greater amplitude changes than the opposite hemisphere when compared with the control condition, and that these shifts would be in the direction of reduced amplitude during task involvement. The present findings support this prediction and are in agreement with those of Galin and Ellis (1975) using spatial and verbal tasks as distractors.

These findings lend further support to the use of the CEP in examining the functional hemispheric asymmetry of the cortex in terms of understanding the nature of its underlying electrical activity.

\section{REFERENCES}

Benton. A. L. The problem of cerebral dominance. Canadian Psychologist, 1965. 6a. 332-348.

Buschbaum. M.. \& Fedio, P. Visual information and evoked responses from the left and right hemispheres. Electroencephalography and Clinical Neurophysiology, 1969. 26. $266-272$.

Butier. S. R., \& Glass. A. Asymmetries in the electroencephalogram associated with cerebral dominance. Electroencephalography and Clinical Neurophysiology. 1974. 36. $4 \times 1-491$.

Ciganek. L. Excitability cycle of the visual cortex in man. Annals of the Ne'l York Academy of Sciences, 1964, 112, 241-253.

(iGANEK. L. The effects of attention and distraction on the visual eroked potential in man: A preliminary report. Electroencephalography and Clinical Neurophysiology. 1965. 18. $625-629$.

CIGANEK. L. Visually evoked potential correlates of attention and distraction in man. Psychiatra Clinica. 1969, 2, 95-108.

СонN. R. Differential cerebral processing of noise and verbal stimuli. Science. 1971, 172, 599-601.

Davis. A. E.. \& WADA. J. Hemispheric asymmetry: Frequency analysis of visual and auditory evoked responses to nonverbal stimuli. Electrocencephalography and Clinical Neurophysiology. 1974, 37. 1.9.

Doyle. J. C. Ornstein. R..\& Galin. D. Lateral specialization of cognitive mode: II. EEG frequency analysis. Psychophysiology, $1974,11.567-578$.

Dumas. R. d Morgan. A. EEG asymmetry as a function of occupation, task, and task difficulty. Neuropsychologia, 1975. 13. $219-228$.
Galin. D.. d Ellis, R. R. Asymmetry in evoked potentials as an index of lateralized cognitive processes: Relation to EEG alpha symmetry. Neuropsychologia, 1975, 13, 45-50.

Galin. D.. \& Ornstein. R. Lateral specialization of cognitive mode: An EEG study. Psychophysiology, 1972, 9, 412-418.

Garcia-Austt, E., Bogacz, J., \& Vanzulli, A. Effects of attention and inattention upon visual evoked response. Electroencephalography and Clinical Neurophysiology, 1964. 17. 136-143.

Haider, M., Spong. P., \& Lindsley, D. B. Attention, vigilance, and cortical evoked-potentials in humans. Science, 1964, 145. 180-182.

JASPER, H. The ten-twenty electrode system of the International Federation. Electroencephulography and Clinical Neurophysiology'. 1958. 10, 371-375.

Lorens, S. A.. JR.. \& Darrow, C. W. Eye movements, EEG, GSR, and EKG during mental multiplication. Electroencephalography and Clinical Neurophysiology, 1902. 14. $739-746$.

McAdam, D. W.. \& Whittaker. H. A. Language production: Electroencephalographic localization in the normal human brain. Science, 1971, 172. 499-502.

McKee, G., Humphrey, B., \& McAdam, D. Scaled lateralization of alpha activity during linguistic and musical tasks. Psychophysiology, 1973, 10. 441-443.

Milner, B. Visually guided maze learning in man: Effects of bilateral, frontal. and unilateral cerebral lesions. Neuropsychologia. 1965. 3. 317-338.

Morgan, A. H.. Macdonald, H., \& Hilgard, E. R. EEG alpha: Lateral asymmetry related to task and hypnotizability. Psychophysiology. 1974. 11. 275-282.

Morgan. A. H., McDonald, P. T., \& Macdonald, H. Differences in bilateral alpha activity as a function of experimental task with a note on lateral eye movements and hypnotizability. Ne'uropsychologia, 1971, 9, 459-469.

Morrell. L. K.. \& Salamy. J. G. Hemispheric asymmetry of electrocortical responses to speech stimuli. Science, 1971, 174. 164-166.

NeVille, H. Electrographic correlates of lateral asymmetry in the processing of verbal and nonverbal auditory stimuli. Journal of Psycholinguistic Research. 1974, 3. 151-163.

SPERRY, R. W. Hemispheric deconnection and unity in conscious awareness. American Psychologist, 1968, 23. 723-733.

Spong, P.. Haider, M.. \& Lindsley, D. B. Selective attentiveness and cortical evoked responses to visual and auditory stimuli. Science. 1965, 148, 395-397.

Taub, J. M.. Tanguay, P. E., Doubleday, C. N., Clarkson, D., \& Remington, R. Hemisphere and ear asymmetry in the auditory evoked response to musical chord stimuli. Physiological Psychology. 1976. 4, 11-17.

(Received for publication July 23, 1977.) 